\title{
Paniculitis lúpica
}

\author{
Lupus panniculitis
}

\section{Luis Fernando González ${ }^{1}$, Yenny Estupiñán Salazar², Fernando Vela ${ }^{3}$, Adriana Motta ${ }^{4}$, Mariam Rolón 5}

1. Médico dermatólogo, programa de Dermatologia, Universidad El Bosque, Bogotá, D.C., Colombia

2. Médico, residente de Dermatología de segundo año, Servicio de Dermatología, Hospital Simón Bolívar, Universidad El Bosque, Bogotá, D.C., Colombia

3. Médico internista, especialista en Reumatología, Universidad Nacional de Colombia, Bogotá, D.C., Colombia

4. Médica dermatóloga, especialista en Epidemiología; jefe, Servicio de Dermatología, Hospital Simón Bolívar, Universidad El Bosque, Bogotá, D.C., Colombia

5. Médica dermatóloga, especialista en Dermatopatología, Servicio de Patología, Hospital Simón Bolívar, Bogotá, D.C., Colombia

\section{RESUMEN}

La paniculitis lúpica es un subtipo del lupus cutáneo de etiología autoinmunitaria que puede estar relacionada con otras formas de lupus cutáneo, lupus sistémico $u$ otras enfermedades autoinmunitarias. Clínica e histológicamente puede confundirse con el linfoma subcutáneo de células T similar a la paniculitis. El tratamiento de este tipo de paniculitis no se ha estandarizado dada la baja prevalencia de la enfermedad.

Se presenta una revisión de los aspectos epidemiológicos, histopatológicos, clínicos y terapéuticos de la paniculitis lúpica.

PALABRAS CLAVE: paniculitis de lupus eritematoso, lupus eritematoso cutáneo, linfoma de células T.

\section{SUMMARY}

Lupus panniculitis is a subtype of cutaneous lupus with an autoimmune etiology that may be related to other forms of cutaneous lupus, SLE and other autoimmune diseases. Clinically and histologically it may be diagnosed as subcutaneous panniculitis-like T-cell lymphoma. Treatment of this type of panniculitis has not been standardized given the low prevalence of the disease.

We review the epidemiological, histopathological, clinical, and therapeutic aspects of lupus panniculitis.

KEY WORDS: Panniculitis, lupus erythematosus; lupus erythematosus, cutaneous; lymphoma, T-cell.

\section{Correspondencia:}

Luis Fernando González

Email:

luisfer10oo@gmail.com

Institución:

Universidad El Bosque, Bogotá, Colombia.

Recibido: 26 de abril de 2016

Aceptado: 5 de agosto de 2016

No se reportan conflictos de interés. 
TABLA 1. Características histopatológicas de la paniculitis lúpica ${ }^{13}$

Criterios mayores

- Necrosis grasa hialina con infiltración linfocítica

- Formación de folículos linfoides

- Paniculitis linfocítica lobular o periseptal

- Calcificación

- Presencia de polvo nuclear dentro del infiltrado (cariorrexis)
Criterios menores

- Cambios de lupus eritematoso discoide

- Inflamación linfocítica vascular

- Hialinización de la zona subepidérmica

- Depósitos de mucina

- Presencia de histiocitos y pequeños granulomas

- Infiltrados de células plasmáticas y eosinófilos

\section{INTRODUCCIÓN}

La paniculitis lúpica o lupus profundo es una variante del lupus eritematoso que afecta el tejido celular subcutáneo; fue descrita por primera vez en 1883 por Kaposi ${ }^{1}$ y relacionada con el lupus eritematoso sistémico en 1940 por Irgang ${ }^{2}$. Más adelante, en 1956, Arnold ${ }^{3}$ publicó cuatro nuevos casos con una revisión de la literatura, donde describió por primera vez la existencia de paniculitis lúpica en ausencia de lupus eritematoso discoide crónico, la cual consideró una variante distinta e independiente del lupus eritematoso. Después de la revisión integral de Arnold, en otros artículos clásicos, Fountain ${ }^{4}$, Winkelmann ${ }^{5}$ y Tuffanelli ${ }^{6}$, finalmente, establecieron las características clínicas, patológicas e inmunológicas de la paniculitis lúpica ${ }^{7}$.

\section{EPIDEMIOLOGÍA}

La paniculitis lúpica es una enfermedad inflamatoria de etiología autoinmunitaria con compromiso del tejido celular subcutáneo que puede relacionarse con otras formas de lupus cutáneo o lupus sistémico. Es un subtipo de paniculitis poco frecuente, presente en $1 \%$ a $3 \%$ de los pacientes con lupus cutáneo ${ }^{8}$. La paniculitis lúpica puede afectar a ambos sexos, aunque es más frecuente en las mujeres con una relación de 4 a 1 en comparación con el sexo masculino ${ }^{7}$. La edad de presentación de la enfermedad se encuentra entre los 20 y los 60 años ${ }^{7}$, con una edad promedio de 41 a 42 años ${ }^{9,10}$; es excepcional en la población pediátrica.

La paniculitis lúpica puede manifestarse como única entidad o puede estar asociada con el lupus eritematoso discoide crónico o el lupus eritematoso sistémico; cuando se asocia con estas enfermedades, puede aparecer al mismo tiempo o puede desarrollarse antes o después de la aparición de las características clínicas propias de estas entidades ${ }^{7}$. La frecuencia de la asociación de paniculitis lúpica con lupus eritematoso discoide crónico es variable. En tres grandes series de casos, esta asociación estaba presente en $21 \%$, $33 \%$ y $60 \%{ }^{6,11,12}$. Lo mismo ocurre con la paniculitis lúpica asociada al lupus eritematoso sistémico, que se ha descrito con proporción variable del $10 \%$ a $4^{2} \%^{6,11,13,14}$. Cuando se presenta paniculitis lúpica en un paciente con lupus eritematoso sistémico, es un marcador de pronóstico favorable ${ }^{11}$.

También, puede estar asociado con otras enfermedades autoinmunitarias, como artritis reumatoide, hepatitis autoinmunitaria, síndrome de Sjögren, colitis ulcerosa, tiroiditis de Hashimoto, anemia hemolítica inmunitaria y púrpura trombocitopénica inmunitaria ${ }^{11}$.

\section{FISIOPATOLOGÍA}

Al parecer el mecanismo fisiopatológico está relacionado con el reclutamiento de linfocitos T en las lesiones subcutáneas, a partir de lesiones de lupus eritematoso cutáneo ${ }^{7}$. Se plantea que el reclutamiento de linfocitos T está mediado por la expresión de interferones de tipo I en la piel, los cuales inducen la producción de proteínas antivirales, como la MxA y la IFI27, al igual que quimiocinas, como la CXCL9 y 10, las cuales son ligandos para el receptor CXCR3 ${ }^{15}$. Lo anterior se traduce en un reclutamiento de linfocitos CXCR3 positivos y células dendríticas que median el proceso inflamatorio local ${ }^{15}$. La infiltración linfocítica del panículo se sustenta por la expresión del marcador interferón de tipo I (MxA) en el tejido adiposo de pacientes con paniculitis lúpica, el cual está asociado con la expresión de IP1o/ CXCL10 y la detección de linfocitos citotóxicos CXCR3+ ${ }^{16}$. Otro tipo de moléculas implicadas en la patogénesis de la paniculitis lúpica son la ezrina, la radixina y la moesina, que contribuyen a la expresión de CD44 y otros receptores en glándulas sudoríparas y sebáceas y en panículo adiposo, lo cual permite la migración del linfocito $\mathrm{T}$ a estos sitios ${ }^{17}$.

También, se ha encontrado una disminución de los niveles de $\mathrm{C}$, lo cual plantea la posibilidad de un componente genético subyacente en la etiología de esta 


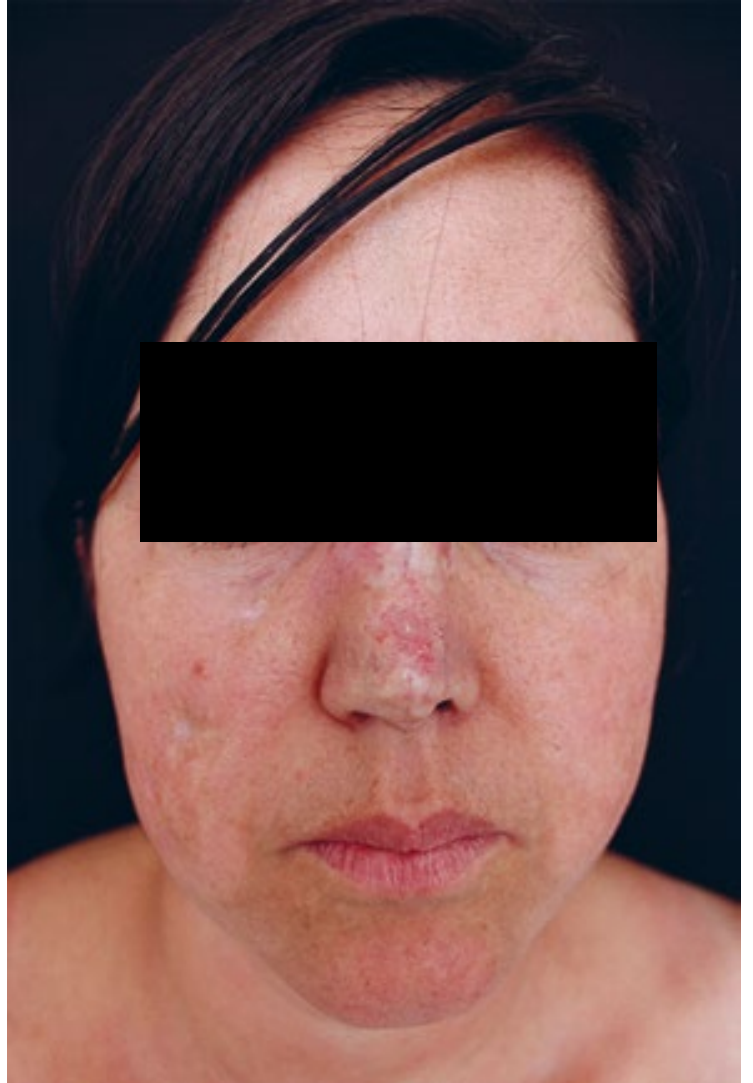

FIgURA 1. Paniculitis lúpica asociada a lupus discoide. Mujer de edad media con placas eritematosas queratósicas que dejan cicatrices atróficas e hipocrómicas en el dorso nasal correspondientes a lupus discoide. En la región malar derecha presenta cicatrices acrómicas con lipoatrofia, histopatología indicaba paniculitis lúpica.

entidad ${ }^{14}$. De igual manera, se ha propuesto que el trauma local puede ser un desencadenante de la enfermedad 7 .

\section{MANIFESTACIONES CLÍNICAS}

La paniculitis lúpica se caracteriza por la presencia de nódulos subcutáneos eritematosos que involucionan dejando áreas lipoatróficas. Compromete principalmente las extremidades proximales, en particular, las caras laterales de los brazos y los hombros ${ }^{7,10}$, y con menor frecuencia, los glúteos, el tronco, la cara y el cuero cabelludo ${ }^{10,18}$ (figuras $1 \mathrm{y}$ 2). El compromiso de los miembros inferiores es inusual y corresponde a una característica clínica importante de otras paniculitis de etiología diferente, como el eritema nudoso y el eritema indurado de Bazin, entre otros ${ }^{19}$. En ocasiones, los pacientes refieren un trauma previo, como inyecciones intramusculares o toma de biopsia ${ }^{20}$.

Cuando se asocia al lupus eritematoso discoide crónico, las lesiones por paniculitis lúpica se pueden acompañar de placas queratósicas con taponamiento folicular, atrofia, despigmentación, telangiectasias o ulceraciones ${ }^{10}$ (figura 1). Esta última característica está presente hasta en $28 \%$ de los pacientes ${ }^{13}$.

Además de estas características clínicas habituales, existen otras variantes clínicas menos comunes o en áreas menos frecuentes, como el tejido adiposo de la mama, también llamado "mastitis lúpica” 21,22; esta consiste en nódulos mamarios de bordes irregulares unilaterales o bilaterales, que tanto clínica como mamográficamente pueden ser mal interpretados como carcinoma de mama, especialmente en aquellos casos en que la mama es el único sitio involucrado. Otras áreas afectadas con baja frecuencia son la región periorbitaria ${ }^{7}$, la grasa periparatiroidea ${ }^{23,24}$ y el lóbulo de la oreja ${ }^{25}$; también, se han descrito lesiones con disposición lineal ${ }^{26,27} \mathrm{o}$ en asociación con dermatofibromas ${ }^{28}$ o hipertricosis ${ }^{29}$.

Los análisis de autoinmunidad son a menudo normales, pero algunas veces los títulos de anticuerpos antinucleares pueden ser positivos y, con menor frecuencia, están presentes los anticuerpos anti-ADN de doble cadena ${ }^{7}$. Otras posibles anomalías de laboratorio incluyen linfopenia, anemia, disminución de los niveles de $\mathrm{C}_{4} \mathrm{y}$ factor reumatoide positivo ${ }^{13}$. La deficiencia de $\mathrm{C}_{4}$ puede favorecer la aparición de paniculitis lúpica, especialmente en los niños, en quienes se recomienda cuantificar los niveles de $\mathrm{C}_{4}{ }^{30,31}$.

\section{DIAGNÓSTICO HISTOPATOLÓGICO}

En el examen de histopatología, las paniculitis se clasifican según la localización del infiltrado inflamatorio en el tejido celular subcutáneo ${ }^{32}$. Existen tres grupos grandes de paniculitis: aquellas que afectan el septo, las que afectan el lóbulo y las que se relacionan con vasculitis de grandes vasos ${ }^{33}$. Generalmente, la paniculitis lúpica forma parte del grupo lobular; sin embargo, algunos autores no aceptan este sistema de clasificación y utilizan el término de "paniculitis mixta” (del septo y lobular) para referirse a la paniculitis por lupus ${ }^{34}$ (figuras 3 y 4). Peters y Su proponen algunos criterios histopatológicos para el diagnóstico de la paniculitis lúpica, dividiéndolos en dos grupos: criterios mayores (importantes para el diagnóstico) y 
criterios menores (no necesarios para el diagnóstico) ${ }^{13,21}$. Los criterios mayores incluyen: necrosis grasa hialina con infiltración linfocítica, formación de folículos linfoides, paniculitis linfocítica lobular o periseptal y calcificación, siendo este último hallazgo el menos frecuente ${ }^{7}$. Entre los criterios menores se encuentran cambios histopatológicos de lupus cutáneo, como degeneración vacuolar de la membrana basal con infiltrado linfoide perivascular superficial y profundo con extensión perifolicular ${ }^{33}$. Otros criterios menores descritos son: inflamación linfocítica perivascular, hialinización de la zona subepidérmica, depósitos de mucina, presencia de histiocitos y pequeños granulomas, e infiltrados de células plasmáticas y eosinófilos (tabla 1). Uno de los hallazgos claves reportado en diferentes series de casos, es la presencia de polvo nuclear linfocítico (cariorrexis) dentro del infiltrado en los lóbulos del tejido adiposo afectado ${ }^{35}$.

La inmunofluorescencia directa puede mostrar una marcación de tipo granular de IgG, IgM y $\mathrm{C}_{3}$ en la

FIGURA 2. Manifestaciones clínicas de la paniculitis lúpica. Nódulos violáceos indurados y dolorosos a la palpación en la región infraescapular y la lumbar izquierda (recuadro). Área lipoatrófica en una paciente con paniculitis lúpica (flecha).

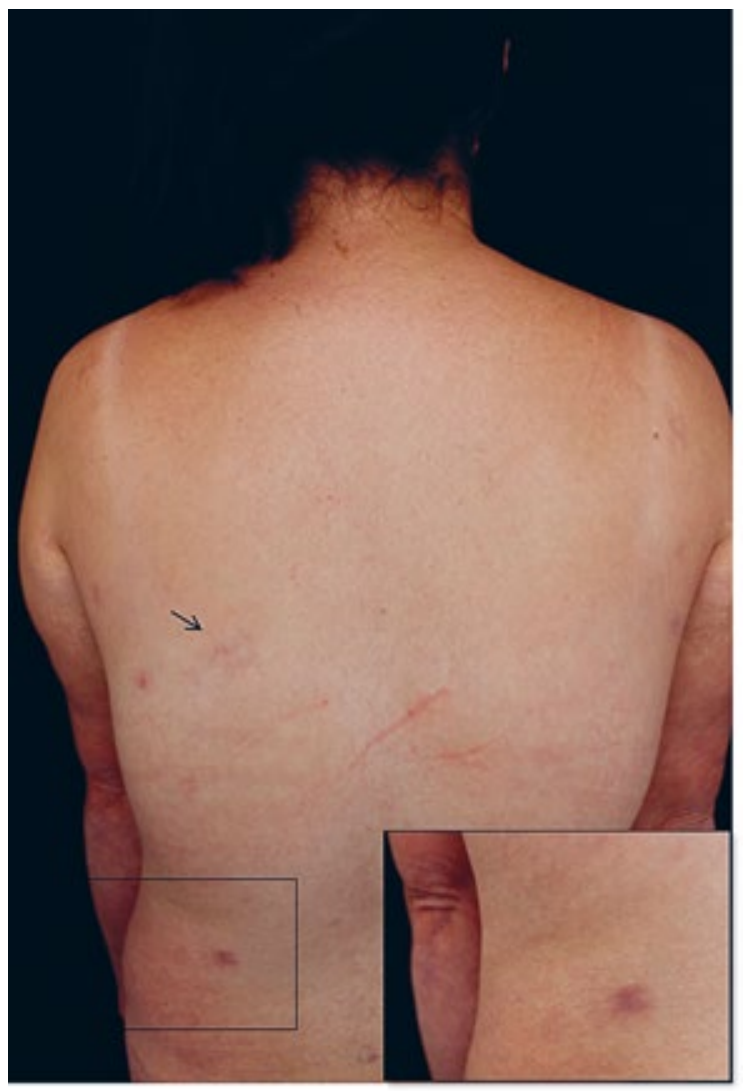

unión dermoepidérmica $\mathrm{y}$, en ocasiones, se pueden encontrar depósitos de complejos inmunológicos en pequeños vasos profundos ${ }^{33}$. El depósito de IgM y $\mathrm{C}_{3}$ en la membrana basal y a nivel perivascular se ha documentado en pacientes con paniculitis lúpica asociada a lupus eritematoso sistémico ${ }^{35}$. La presencia de banda lúpica positiva se ha observado en 70 a $90 \%$ de los pacientes con paniculitis lúpica no asociada a enfermedad sistémica ${ }^{5-7}$, y es de utilidad para la confirmación de la esta entidad.

\section{DIAGNÓSTICO DIFERENCIAL}

Como integrante del amplio grupo de enfermedades inflamatorias del tejido adiposo subcutáneo, la paniculitis lúpica guarda ciertas similitudes con otras paniculitis y, con mayor relevancia, con el subgrupo de las paniculitis lobulares ${ }^{33}$. En este subgrupo, el principal diagnóstico diferencial debe hacerse con el linfoma

FigURA 3. Biopsia de piel y tejido celular subcutáneo en la cual se destaca una paniculitis linfocítica de predominio lobulillar, sin vasculitis. Hematoxilina y eosina, $20 X$.

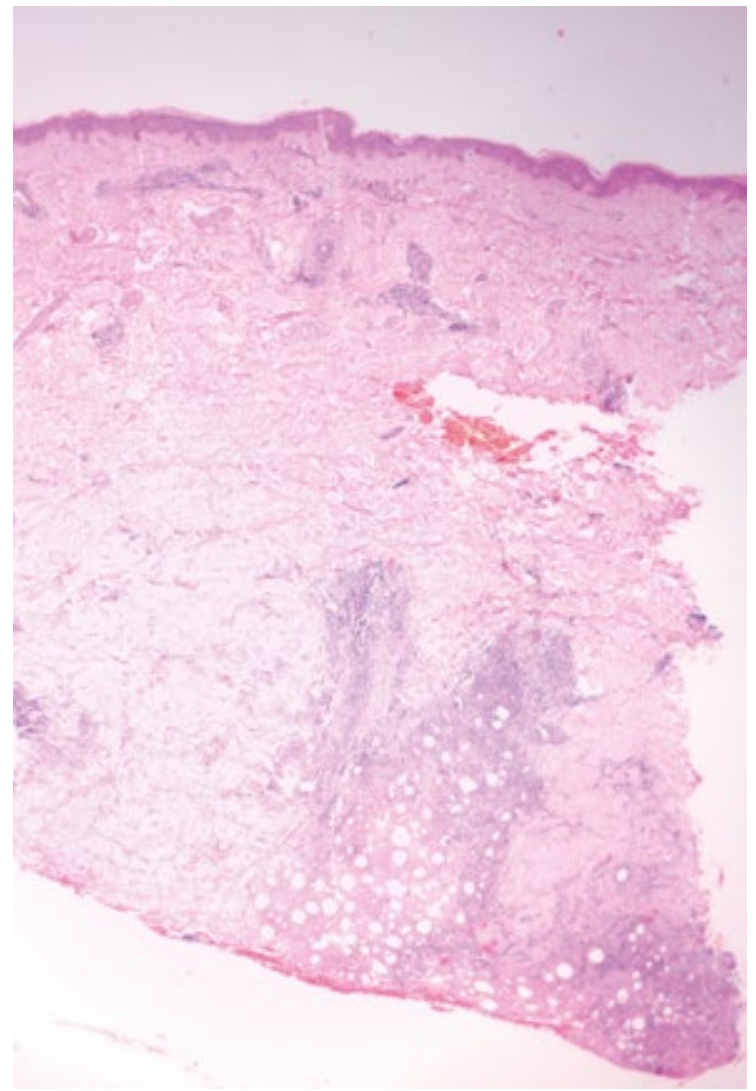


subcutáneo de células T similar a paniculitis (Subcutaneous Panniculitis-Like T-cell Lymphoma, SPTCL), en el cual la histopatología resulta fundamental para determinar las diferencias entre las dos entidades. En la paniculitis lúpica se observa compromiso epidérmico, folículos linfoides con centros germinales reactivos e infiltrado mixto de células (con predominio de células plasmáticas) ${ }^{33}$. Mientras que, en el linfoma, se pueden encontrar linfocitos atípicos con marcadores inmunohistoquímicos de linaje $\mathrm{T}\left(\mathrm{CD}_{3}+, \mathrm{CD} 8+\right)$ y marcadores citotóxicos (CD56, Granzima, TIA y perforina), acompañados de un índice de proliferación aumentado (Ki-67) y rearreglos genéticos monoclonales TCR-gamma ${ }^{36}$. Además, en algunos estudios comparativos, se ha encontrado que los conglomerados de células dendríticas plasmocitoides identificadas por inmunohistoquímica con el marcador CD123 son específicos de paniculitis lúpica y no de linfoma subcutáneo de células T similar a paniculitis ${ }^{7,37}$.

Otras paniculitis lobulares con las que se debe hacer diagnóstico diferencial, son el eritema indurado de
Bazin (vasculitis nodular) y la paniculitis pancreática. La paniculitis lúpica es menos granulomatosa y tiene un mayor infiltrado linfoplasmocelular en comparación con el eritema indurado, el cual se asocia a un importante fenómeno de vasculitis ${ }^{35}$. Por otra parte, en la paniculitis pancreática hay un patrón de necrosis basofílica homogénea o granular, mientras que en la paniculitis lúpica se pueden presentar depósitos de mucina con el característico infiltrado linfoplasmocelular y los cambios dermoepidérmicos propios del lupus eritematoso ${ }^{37}$.

Otras entidades que pueden acompañarse con cambios del tejido celular subcutáneo o cursar con una paniculitis propia, son la morfea y la dermatomiositis con paniculitis asociada, en las cuales se encuentran folículos linfoides con menor frecuencia que en la paniculitis lúpica ${ }^{37}$. Si bien el CD123 se ha observado en las células dendríticas plasmocitoides en algunos pacientes con paniculitis lúpica, este marcador se puede expresar en otras enfermedades que afectan el tejido celular subcutáneo, como la paniculitis inducida por frío ${ }^{37}$.

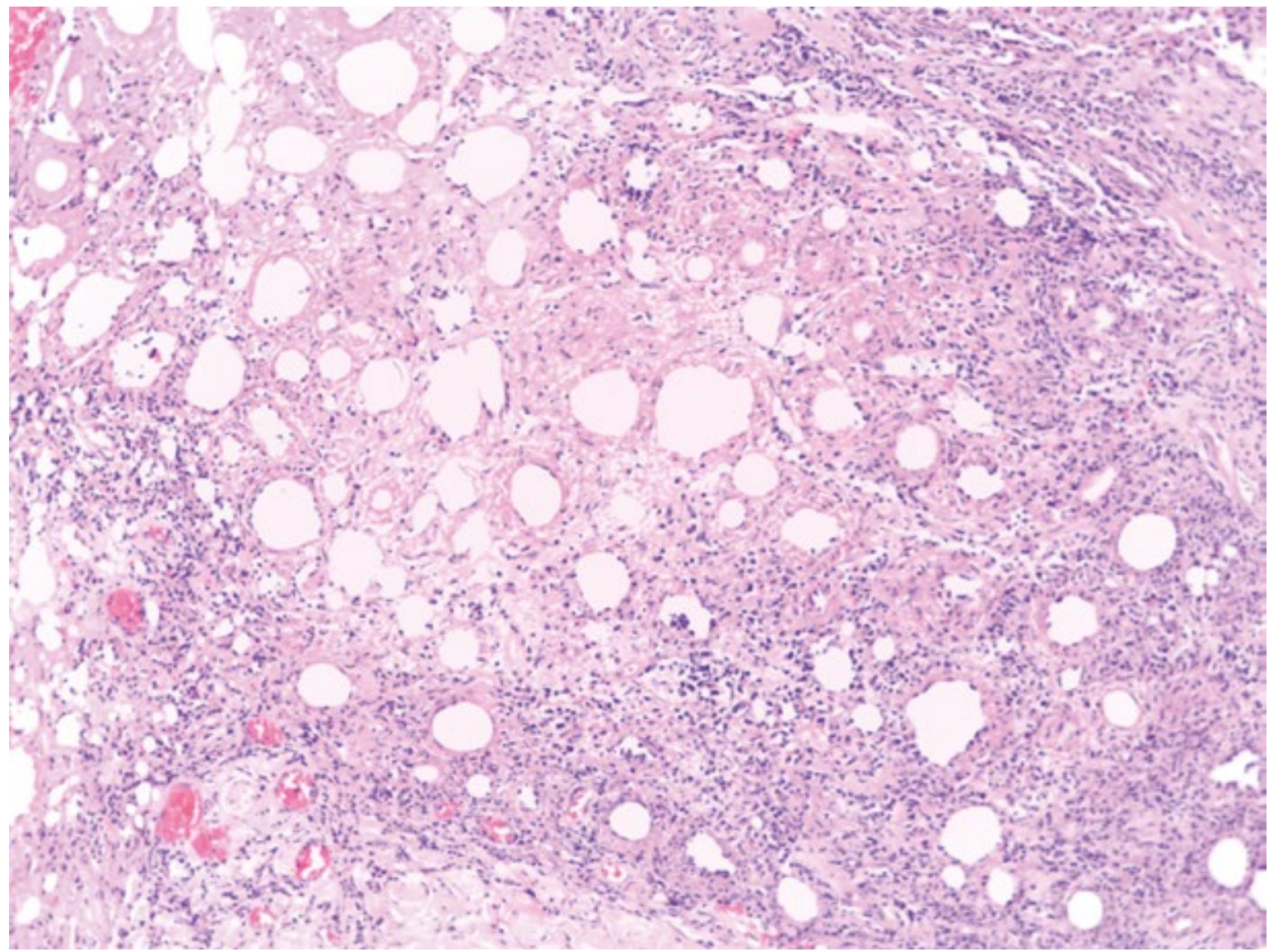

FIGURA 4. Áreas de necrosis fibrinoide que rodean lobulillos del tejido celular subcutáneo con infiltrado linfoide. hematoxilina y eosina, 40X. 


\section{TRATAMIENTO}

El tratamiento de la paniculitis lúpica es complejo de estandarizar, debido a su baja prevalencia y la historia natural de la enfermedad caracterizada por frecuentes recaidas y lesiones persistentes ${ }^{38}$. De igual manera, es complejo establecer su actividad clínica a pesar de contar con herramientas validadas como el índice de severidad y áreas de lupus cutáneo (ISALC), el cual tiene poca utilidad en el lupus profundo ${ }^{39}$. Sin embargo, en 2010 se revisó el ISALC para ajustar la precisión de los parámetros usados, creando así el "ISALC revisado", que actualmente se aplica en diferentes estudios para la evaluación clínica de los subtipos de lupus eritematoso cutáneo ${ }^{40}$.

A pesar del advenimiento de nuevas terapias farmacológicas en enfermedades autoinmunitarias, no se ha modificado la de la paniculitis lúpica: los antipalúdicos se siguen considerando la primera línea del tratamiento de lesiones en adultos y en niños ${ }^{38}$. Básicamente, la evidencia disponible se limita a reportes de casos, series de casos y algunos estudios prospectivos en los que los pacientes con paniculitis lúpica representan la minoría.

\section{PRIMERA LÍNEA}

\section{Antipalúdicos}

El mecanismo de acción de los antipalúdicos no se ha elucidado completamente, aunque su efecto posiblemente se debe a la acción inmunomoduladora al alterar la presentación de antígenos, la estabilización de lisosomas y la supresión de la transducción de la señal por los receptores de tipo toll (especialmente TLR9) ${ }^{41}$. Se prefiere la hidroxicloroquina como monoterapia frente a la cloroquina, por su perfil farmacológico más seguro y su menor toxicidad en la retina ${ }^{39}$. La dosis recomendada de hidroxicloroquina es de $200 \mathrm{mg}$, dos veces al día, con una dosis diaria máxima de $6,5 \mathrm{mg} / \mathrm{kg}$; su acción inicia en cuatro a ocho semanas, aunque se ha reportado respuesta clínica después de seis meses de iniciado este medicamento. La cloroquina como monoterapia se recomienda a dosis de 250 a $500 \mathrm{mg}$ al día, evitando dosis diarias mayores de $3,5 \mathrm{mg} / \mathrm{kg}{ }^{39}$. Dado el riesgo de retinopatía, se recomienda el control anual por oftalmología y, con mayor frecuencia, en pacientes mayores de 50 años o con enfermedad renal o hepática ${ }^{40}$.
Otros efectos adversos comunes de los antipalúdicos incluyen molestias gastrointestinales y cambios en la piel, como erupciones medicamentosas liquenoides, prurito y alteraciones de la pigmentación ${ }^{42}$. Los efectos adversos raros incluyen toxicidad hematológica, psicosis, miopatía y cardiomiopatía. En algunos casos cuando las lesiones presentan nódulos calcificados, pueden beneficiarse de la combinación con diltiazem ${ }^{42}$.

\section{Corticoides orales}

Estos son eficaces para el manejo de los ataques agudos por lupus eritematoso sistémico y algunos casos del discoide crónico, así como de otras formas de lupus cutáneo, entre ellas la paniculitis lúpica. La administración de corticoesteroides resulta en una rápida depleción de células T circulantes debido a una combinación de efectos que incluyen aumento de la emigración circulatoria, inducción de apoptosis, inhibición de los factores de crecimiento de células $\mathrm{T}$ y alteración en la liberación de células desde los tejidos linfoides ${ }^{43}$. Todo lo anterior se traduce en un efecto inmunosupresor importante con riesgo de infecciones y predisposición a complicaciones, como osteoporosis, síndrome de Cushing, osteonecrosis, hipertensión arterial sistémica, hiperglucemia y aumento del proceso de la aterogénesis cuando son usados por tiempos prolongados, como ocurre en estos pacientes ${ }^{42,44}$. La dosis diaria de corticoesteroides en el lupus cutáneo es de 0,5 a $2 \mathrm{mg} / \mathrm{kg}$ por dos a cuatro semanas ${ }^{42}$. Sin embargo, según la evidencia disponible, se recomienda el uso de corticoesteroides orales en los casos más graves asociados a lupus eritematoso sistémico, debido a la morbilidad del uso prolongado, incluyendo la atrofia de las lesiones ${ }^{40}$.

\section{SEGUNDA LÍNEA}

\section{Corticoides tópicos}

Al igual que los corticoides sistémicos, estos actúan sobre la transcripción genética de células inflamatorias, principalmente el linfocito $\mathrm{T}{ }^{44}$. Existen reportes del uso tópico de corticoides de alta potencia, como el propionato de clobetasol al 0,05\% en vendaje oclusivo ${ }^{44}$; sin embargo, las publicaciones sobre su uso son escasas. Por otra parte, los esteroides intralesionales generalmente son ineficaces y aumentan la atrofia, por lo cual no se recomiendan ${ }^{7}$. 


\section{Segundo agente antipalúdico}

La quinacrina fue usada en la década de $1940 \mathrm{du}$ rante la segunda guerra mundial como terapia profiláctica para la malaria y se evidenció mejoría clínica de los soldados con lupus o artritis reumatoide al recibir el tratamiento ${ }^{45}$. Sin embargo, no fue sino hasta 1951 cuando Page ${ }^{46}$ presentó la primera serie de pacientes con lupus discoide con buena respuesta terapéutica a la quinacrina. Su uso se limitó por complicaciones graves como la anemia aplásica, la cual puede presentarse en uno de cada 500.000 pacientes ${ }^{46}$. La quinacrina se usa a dosis máximas de $100 \mathrm{mg} /$ día y se reserva para aquellos pacientes con antecedentes de toxicidad retiniana por hidroxicloroquina o cloroquina. No obstante, su asociación con otros agentes antipalúdicos es útil para tratar las lesiones de paniculitis lúpica ${ }^{47}$.

\section{Talidomida}

El uso de la talidomida en enfermedades inflamatorias se debe al efecto inmunomodulador y la actividad antiTNF-alfa, sumado a sus propiedades antiangiogénicas ${ }^{48}$. La talidomida es una opción efectiva para la paniculitis lúpica; sin embargo, se considera como una alternativa de segunda línea en los pacientes que no han mejorarado con los antipalúdicos y corticoides orales, debido a efectos adversos como la teratogenicidad, la neuropatía periférica y la trombosis ${ }^{40,49,50}$. Se cree que la continuación del tratamiento con antipalúdicos por su efecto antiplaquetario o el uso de aspirina pueden mitigar el efecto trombótico de la talidomida, aunque no existen guías sobre tromboprofilaxis para los pacientes que reciben este fármaco $4^{\circ}$.

La mejoría de las lesiones de paniculitis mediante el tratamiento con talidomida se ha documentado en varios artículos. En 60 casos de lupus cutáneo resistente al tratamiento, se estudió prospectivamente el efecto de $100 \mathrm{mg}$ diarios de talidomida en el control de la enfermedad ${ }^{51}$. En esta cohorte, cinco pacientes recibieron talidomida para la paniculitis lúpica con mejorías completa o parcial, en un tiempo promedio de seguimiento de nueve meses ${ }^{52}$. La dosis de talidomida osciló entre 50 y $300 \mathrm{mg}$ diarios, aunque generalmente no se necesitan dosis superiores a $150 \mathrm{mg}^{40,42}$.

Otros efectos adversos, como somnolencia, estreñimineto, cefalea, aumento de peso y amenorrea, están relacionados con la dosis usada ${ }^{40}$. Se recomienda usar la dosis más baja posible, incluyendo $100 \mathrm{mg}$ en días alternos o $50 \mathrm{mg}$ diarios, como esquemas de mantenimiento ${ }^{26}$.
La lenalidomida es un análogo de la talidomida usado en el manejo del mieloma múltiple, con efectos similares a los de la talidomida en el tratamiento de otros tipos de lupus cutáneo; sin embargo, no se ha reportado su uso en paniculitis lúpica ${ }^{52}$.

\section{Dapsona}

La dapsona tiene propiedades antiinflamatorias y antibióticas al inhibir la dihidrofolato reductasa ${ }^{42}$. Se ha utilizado en Japón, donde la hidroxicloroquina y la talidomida no están disponibles. En una revisión de 10 casos de paniculitis lúpica tratados con dosis entre 25 y 75 mg diarios, la remisión se logró después de un tiempo variable de una a ocho semanas ${ }^{53}$. Tres pacientes experimentaron efectos adversos moderados, como erupciones medicamentosas, cefalea, hipertensión arterial sistémica y anemia ${ }^{53}$. La dapsona puede causar efectos adversos dependientes de la dosis tan graves como la anemia hemolítica y la metahemoglobinemia ${ }^{42}$. Por lo tanto, en los pacientes que van a recibir tratamiento con dapsona debe descartarse deficiencia de glucosa-6-fosfato reductasa ${ }^{40,42}$. Además, se puede presentar agranulocitosis idiosincrática o reacciones de hipersensibilidad, por lo cual es importante practicar regularmente exámenes de hemograma y de función hepática ${ }^{42}$.

\section{TERCERA LÍNEA}

\section{Inmunoglobulina intravenosa}

El mecanismo de acción de la inmunoglobulina intravenosa en las enfermedades autoinmunitarias no se conoce completamente, aunque se plantea que interviene en la regulación negativa de los linfocitos $\mathrm{B}$ autorreactivos ${ }^{54}$ y en la inhibición de la citotoxicidad mediada por células dependientes de anticuerpos, y se cree que aumenta el efecto de las células T reguladoras, por medio del incremento de la IL 10 y el factor de crecimiento transformante beta ${ }^{55}$. Existe un solo reporte de caso sobre el uso de la inmunogobulina intravenosa en paniculitis lúpica resistente al tratamiento convencional, en el cual se plantea como una alternativa terapéutica capaz de controlar y mantener el estado de remisión sin generar reacciones adversas agresivas por inmunosupresión ${ }^{56}$. La dosis total usada fue de $2 \mathrm{~g} / \mathrm{kg}$ distribuidos en cinco días (400 mg/kg diarios administrados a menos de $50 \mathrm{mg} /$ 
kg por hora) y en un tiempo mayor de ocho horas para minimizar los riesgos ${ }^{57}$.

\section{Rituximab}

El rituximab es un anticuerpo monoclonal quimérico que inhibe los linfocitos $\mathrm{B}$ al unirse con el marcador de superficie $\mathrm{CD}_{20}{ }^{57}$. Su uso fue aprobado para el manejo de linfomas de células $\mathrm{B}$ y de artritis reumatoide resistente; sin embargo, ha sido ampliamente usado en diferentes condiciones dermatológicas, entre ellas, la paniculitis lúpica ${ }^{58,59}$. Existen dos reportes ${ }^{57,58}$ en los que se exponen tres pacientes con paniculitis lúpica resistente al tratamiento convencional, quienes presentaron una respuesta clínica satisfactoria. Los efectos adversos agudos fueron mínimos, entre los cuales se destaca la cefalea y la fatiga ${ }^{58}$, aunque no se mencionan complicaciones posteriores ni seguimiento a largo plazo.

\section{Ciclosporina}

La ciclosporina A bloquea la actividad de la calcineurina fosfatasa, inhibiendo la activación de los linfocitos $\mathrm{T}$ y B ${ }^{42}$. La ciclosporina es poco usada para el lupus cutáneo debido a su efectividad moderada y al compromiso de la función renal ${ }^{60}$. Se ha usado en pacientes con paniculitis lúpica y lupus eritematoso sistémico de presentación aguda, con remisión rápida ${ }^{61}$. De igual manera, es una opción válida en los pacientes con paniculitis lúpica resistente al tratamiento y como alternativa de mantenimiento del tratamiento ${ }^{61,62}$.

\section{OTRAS OPCIONES TERAPÉUTICAS}

\section{Terapia biológica con anti-TNF}

El advenimiento de la terapia biológica anti-TNF ha revolucionado el manejo de enfermedades inflamatorias de la piel como la psoriasis; sin embargo, su uso también se ha orientado al manejo de entidades como el lupus eritematoso sistémico, siendo excepcional su uso en el lupus cutáneo. El infliximab se ha usado como tratamiento coadyuvante en casos de paniculitis lúpica grave asociados a lupus eritematoso sistémico. En un paciente con paniculitis lúpica, el tratamiento con infliximab asociado a metotrexato e hidroxicloroquina garantizó el control y la remisión de la enfermedad en un periodo de 12 meses $^{63}$. Si bien la terapia anti-TNF puede ser usada en casos resistentes y particulares, también se ha documentado inducción de paniculitis lúpica después de su administración ${ }^{64}$.

\section{Otros inmunosupresores}

Existen diferentes casos anecdóticos del uso de medicamentos como metotrexato, azatioprina y micofenolato de mofetilo en el lupus cutáneo ${ }^{65}$. El metotrexato se usó en un paciente con paniculitis lúpica resistente al tratamiento con corticoesteroides sistémicos, a una dosis de $25 \mathrm{mg}$ semanales durante siete semanas, con respuesta clínica favorable y sin reactivación durante seis meses ${ }^{66}$.

\section{Transferencia autóloga de grasa}

Se plantea que la transferencia autóloga de grasa es una opción terapéutica en esta enfermedad, ya que se ha considerado que los adipocitos pueden ejercer funciones de células madre y estimular el colágeno, estabilizando así el medio local y aumentando el volumen del tejido circundante ${ }^{66}$.

\section{Materiales de relleno}

Existe preocupación sobre el uso de materiales de relleno en enfermedades del tejido conjuntivo, debido al riesgo teórico de exacerbación de la enfermedad como resultado de la activación antigénica. Sin embargo, no se ha documentado tal efecto en rellenos inertes, como el ácido poli-L-láctico o el ácido hialurónico, los cuales demostraron efectividad en la lipoatrofia facial localizada en una paciente con paniculitis lúpica ${ }^{67}$.

\section{Fotoprotección}

La radiación ultravioleta es reconocida como un desecadenante ambiental importante para el lupus cutáneo. Si bien no es claro su papel en la paniculitis lúpica, al igual que en otras formas de lupus cutáneo, se recomienda el uso diario de protector solar ${ }^{7,40}$. 


\section{"Las alternativas terapéuticas disponibles para el tratamiento de la paniculitis lúpica se basan en reportes de caso y opinión de expertos, y son un desafío para el dermatólogo".}

\section{CONCLUSIONES}

La paniculitis lúpica representa una variante poco frecuente del lupus cutáneo, caracterizada por el compromiso del tejido celular subcutáneo con infiltrado linfocitario perilobulillar, principalmente.

Clínicamente, se presenta como placas eritematosas infiltradas que pueden dejar áreas lipoatróficas deprimidas con consecuencias estéticas desfavorables, sobre todo en las lesiones en el rostro. En la histopatología, el diagnóstico diferencial más importante y difícil se hace con el linfoma subcutáneo de células T de tipo paniculitis.

Las alternativas terapéuticas disponibles para el tratamiento de la paniculitis lúpica se basan en reportes de caso y opinión de expertos, y son un desafío para el dermatólogo. La mejor evidencia disponible sugiere como primera línea de tratamiento a los antipalúdicos y los corticoides orales, seguidos de la talidomida y la dapsona como alternativas para los pacientes con paniculitis lúpica resistente al tratamiento.

\section{REFERENCIAS}

1. Arnold HL.Lupus erythematosus profundus (Kaposi-Irgang) historical review and report of a case. Arch Derm Syphilol. 1948;57:196-203

2. Irgang S. Lupus erythematosus profundus: Report of an example with clinical resemblance to Darier-Roussy sarcoid. Arch Dermat Syph. 1940;42:97-108.

3. Arnold HL. Lupus erythematosus profundus; commentary and report of four more cases. AMA Arch Derm. 1956;73:15-33.

4. Fountain RB. Lupus erythematosus profundus. Br J Dermatol. 1968;80:571-9.

5. Winkelmann RK. Panniculitis and systemic lupus erythematosus. JAMA. 1970;211:472-5.
6. Tuffanelli DL. Lupus erythematosus panniculitis (profundus): Clinical and immunologic studies. Arch Dermatol. 1971;103:231-42.

7. Fraga J, García-Díez A. Lupus erythematosus panniculitis. Dermatol Clin. 2008;26:453-63.

8. Massone C, Kodama K, Salmhofer W, Abe R, Shimizu H, Parodi A, et al. Lupus erythematosus panniculitis (lupus profundus): Clinical, histopathological, and molecular analysis of nine cases. J Cutan Pathol. 2005;32:396-404.

9. Martens PB, Moder KG, Ahmed I. Lupus panniculitis: Clinical perspectives from a case series. J Rheumatol. 1999;26:68-72.

10. De la Moneda Herrerin C, Conde Zurita JM, Guerra Tapia A. El lupus paniculitis: una paniculitis mixta. Actas Dermosifiliogr. 1987;78:229-38.

11. Peters MS, Su WP. Lupus erythematosus panniculitis. Med Clin North Am. 1989;73:1113-25.

12. Tuffanelli DL. Lupus erythematosus (panniculitis) profundus: A classic revisited commentary and report of 22 cases. Hawaii Med J. 1992;41:394-7.

13. Magro CM, Crowson AN, Kovatich AJ, Burns F. Lupus profundus, indeterminate lymphocytic lobular panniculitis and subcutaneous T-cell lymphoma: A spectrum of subcuticular T-cell lymphoid dyscrasia. J Cutan Pathol. 2001;28:235-47.

14. Nousari HC, Kimyai-Asadi A, Provost TT. Generalized lupus erythematosus profundus in a patient with genetic partial deficiency of C4. J Am Acad Dermatol. 1999;41:362-4.

15. Wenzel J, Tüting T. Identification of type I interferonassociated inflammation in the pathogenesis of cutaneous lupus erythematosus opens up options for novel therapeutic approaches. Exp Dermatol. 2007;16:454-63.

16. Wenzel J, Proelss J, Wiechert A, Zahn S, Bieber T, Tüting T. CXCR3-mediated recruitment of cytotoxic lymphocytes in lupus erythematosus profundus. J Am Acad Dermatol. 2007;56:648-50.

17. Abreu-Velez AM, Loebl AM, Howard MS. Autoreactivity to sweat and sebaceous glands and skin homing $\mathrm{T}$ cells in lupus profundus. Clin Immunol. 2009;132:420-4.

18. Tuffanelli DL. Lupus panniculitis. Semin Dermatol. 1985;4:79-81.

19. Patterson JW. Panniculitis. En: Bologna JL, Jorizzo JL, Rapini RP, editores. Dermatology. Londres: Mosby; 2010. p. 1653-4. 
20. Klein SA, Tomaro AJ. Lupus erythematosus profundus: Review of the literature and report of case. J Oral Surg. 1975;33:454-7.

21. Carducci M, Mussi A, Lisi S, Muscardin L, Solivetti FM. Lupus mastitis: A 2-year history of a single localization of lupus erythematosus mimicking breast carcinoma. J Eur Acad Dermatol Venerol. 2005;19:260-2.

22. Sabaté JM, Gómez A, Torrubia S, Salinas T, Clotet M, Lerma E. Lupus paniculitis involving the breast. Eur Radiol. 2006;16:53-6.

23. White WL, Sherertz EF, Berg D, Clark RE. Periparotid lupus erythematosus panniculitis: Clinic pathologic correlation of two cases presenting as primary parotid disease. Arch Pathol Lab Med. 1993;117:535-9.

24. Ogura N, Fujisaku A, Jodo S, Ichikawa K, Tsutsumi A, Mukai $\mathrm{M}$, et al. Lupus erythematosus profundus around the salivary glands: A case resembling submandibular gland disease. Lupus. 1997;6:477-9.

25. Sardana K, Mendiratta V, Koranne RV, Verma R, Vig R. Lupus erythematosus profundus involving the ear lobe. J Eur Acad Dermatol Venerol. 2003;17:727-9.

26. Tamada Y, Arisawa S, Ikeya T, Yokoi T, Hara K, Matsumoto Y. Linear lupus erythematosus profundus in a young man. Br J Dermatol. 1999;140:177-8.

27. Nagai Y, Ishikawa O, Hattori T, Ogawa T. Linear lupus erythematosus profundus on the scalp following the lines of Blaschko. Eur J Dermatol. 2003;13:294-6.

28. Chan I, Robson A, Mellerio JE. Multiple dermatofibromas associated with lupus profundus. Clin Exp Dermatol. 2005;30:128-30.

29. García-Doval I, Roson E, Abalde M, Feal C, Cruces MJ. Coexistence of acquired localized hypertrichosis and lipoatrophy after lupus panniculitis. J Am Acad Dermatol. 2004;50:799-800.

30. Nousari HC, Kimyai-Asadi A, Provost TT.Generalized lupus erythematosus profundus in a patient with genetic partial deficiency of C4. J Am Acad Dermatol. 1999;41:362-4.

31. Cribier B. Panniculite lupique. Presse Med. 2005;34:243-8.

32. Patterson JW. Panniculitis. En: Weedon D, Geoffrey S, Adam IR, editors. Weedon's Skin Pathology. Edinburgh: Churchill Livingstone/Elsevier, 2010. p. 542-3

33. Patterson JW. Differential diagnosis of panniculitis. Adv Dermatol. 1991;6:309-29.

34. Barnhill RL, Crowson AN. Panniculitis. Textbook of Dermatopathology. 2nd edition. New York: McGraw-Hill; 2004. p. 267-98.

35. Arai S, Katsuoka K. Clinical entity of lupus erythematosus panniculitis/lupus erythematosus profundus. Autoimmunity Reviews. 2009;8:449-52.

36. Luzar C. Inflammatory diseases of cutaneous fat: Lupus erythematosus profundus. En: Calonje E, Brenn T, Lazar A, McKee PH, editors. McKee's pathology of the skin: with clinical correlations. 4th ed. Edinburgh: Elsevier/Saunders; 2012. p. 358-360.

37. Hansen CB, Callen JP. Connective tissue panniculitis: Lupus panniculitis, dermatomyositis, morphea/scleroderma. Dermatol Ther. 2010;23: 341-9.

38. Cusack CA, Fanelli M. Panniculitis. En: Lebwohl MG, Heymann WR, Berth-Jones J, eitors. Treatment of Skin Diseases: Comprehensive Therapeutic Strategies. Fourt edition. Philaderphia: Saunders; 2013. p. 528-9.
39. Braunstein I, Werth VP. Update on management of connective tissue panniculitides. Dermatol Ther. 2012;25:173-82.

40. Kuhn A, Landmann A. The classification and diagnosis of cutaneous lupus erythematosus. J Autoimmun. 2014;48-49:14-9.

41. Ziemer M, Milkova L, Kunz M. Lupus erythematosus. Part II: Clinical picture, diagnosis and treatment. J Dtsch Dermatol Ges. 2014;12:285-301.

42. Morgan KW, Callen JP. Calcifying lupus panniculitis in a patient with subacute cutaneous lupus erythematosus: Response to diltiazem and chloroquine. J Rheumatol. 2001;28:2129-32.

43. Chatham WW, Kimberly RP. Treatment of lupus with corticosteroids. Lupus. 2001;10:140-7.

44. Yell JA, Burge SM. Lupus erythematosus profundus treated with clobetasol propionate under a hydrocolloid dressing. $\mathrm{Br}$ J Dermatol. 1993;128:103.

45. González-Sixto B, García-Doval I, Oliveira R, Posada C, García-Cruz MA, Cruces M. Quinacrine in the treatment of cutaneous lupus erythematosus: Practical aspects and a case series. Actas Dermosifiliogr. 2010;101:54-8.

46. Page F. Treatment of lupus erythematosus with mepacrine. Lancet. 1951;2:755-8.

47. Chung HS, Hann SK. Lupus panniculitis treated by a combination therapy of hydroxychloroquine and quinacrine. J Dermatol. 1997;24:569-72.

48. Yang CS, Kim C, Antaya R. Review of thalidomide use in the pediatric population. J Am Acad Dermatol. 2015;72:703-11.

49. Wienart S, Gadola S, Hunziker T. Facets of lupus erythematosus: Panniculitis responding to thalidomide. J Dtsch Dermatol Ges. 2008;6:214-6.

50. Burrows NP, Walport MJ, Hammond AH, Davey N, Jones RR. Lupus erythematosus profundus with partial $\mathrm{C}_{4}$ deficiency responding to thalidomide. Br J Dermatol. 1991;125:62-7.

51. Hansen CB, Dahle KW. Cutaneous lupus erythematosus. Dermatol Ther. 2012;25:99-111.

52. Cortés-Hernández J, Torres-Salido M, Castro-Marrero J, Vilardell-Tarres M, Ordi-Ros J. Thalidomide in the treatment of refractory cutaneous lupus erythematosus: Prognostic factors of clinical outcome. Br J Dermatol. 2012;166:616-23.

53. Ujiie H, Shimizu T, Ito M, Arita K, Shimizu H. Lupus erythematosus profundus successfully treated with dapsone: Review of the literature. Arch Dermatol. 2006:142:399-401.

54. Samuelsson A, Towers TL, Ravetch JV. Anti-inflammatory activity of IVIG mediated through the inhibitory Fc receptor. Science. 2001;291:484

55. Fehr J, Hoffman V, Kappeler U. Transient reversal of thrombocytopenia in idiopathic thrombocytopenic purpura by high-dose intravenous gamma globulin. N Engl J Med. 1982;306:1254.

56. Santo JE, Gomes MF, Gomes MJ, Peixoto LC, Pereira S, Acabado A, et al. Intravenous immunoglobulin in lupus panniculitis. Clin Rev Allergy Immunol. 2010;38:307-18.

57. Moreno-Suárez F, Pulpillo-Ruiz A. Rituximab for the treatment of lupus erythematosus panniculitis. Dermatol Ther. 2013;26:415-8.

58. McArdle A, Baker JF. A case of "refractory" lupus erythematosus profundus responsive to rituximab. ClinRheumatol. 2009;28:745-6.

59. Wozniacka A, Salamon M, Lesiak A, McCauliffe DP, Sysa-Jedrzejowska A. The dynamism of cutaneous lupus 
erythematosus: Mild discoid lupus erythematosus evolving into SLE with SCLE and treatment resistant lupus panniculitis. Clin Rheumatol. 2007;26:1176-9.

6o. Kuhn A, Ruland V, Bonsmann G. Cutaneous lupus erythematosus: Update of therapeutic options. Part II. J Am Acad Dermatol. 2011;65:195-213.

61. Saeki Y, Ohshima S, Kurimoto I, Miura H, Suemura M. Maintaining remission of lupus erythematosus profundus (LEP) with cyclosporin A. Lupus. 2000;9:390-2.

62. Ishiguro N, Iwasaki T, Kawashima M, Kawakami M. Intractable ulceration in a patient with lupus erythematosus profundus successfully treated with cyclosporine. Int J Dermatol. 2012; 51:1131-3.

63. Günther C, Aringer M, Lochno M, Kämmerer E, Bauer A, Wozel G, et al. TNF-alpha blockade with infliximab in a patient with lupus erythematosus profundus. Acta Derm Venereol. 2012;92:401-3.

64. Lee H, Kim DS, Chung KY. Adalimumab-induced lupus panniculitis. Lupus. 2014;23:1443-4.

65. Winkelmann RR, Kim GK, Del Rosso JQ. Treatment of cutaneous lupus erythematosus: Review and assessment of treatment benefits based on Oxford Centre for evidencebased medicine. J Clin Aesthet Dermatol. 2013;6:27-38.

66. Boehm IB, Boehm GA, Bauer R. Management of cutaneous lupus erythematosus with low-dose methotrexate: Indication for modulation of inflammatory mechanisms. Rheumatol Int. 1998;18:59-62.

67. Eastham AB, Liang CA, Femia AN, Lee TC, Vleugels RA, Merola JF. Lupus erythematosus panniculitis-induced facial atrophy: effective treatment with poly-L-lactic acid and hyaluronic acid dermal fillers. J Am Acad Dermatol. 2013;69:260-2. 